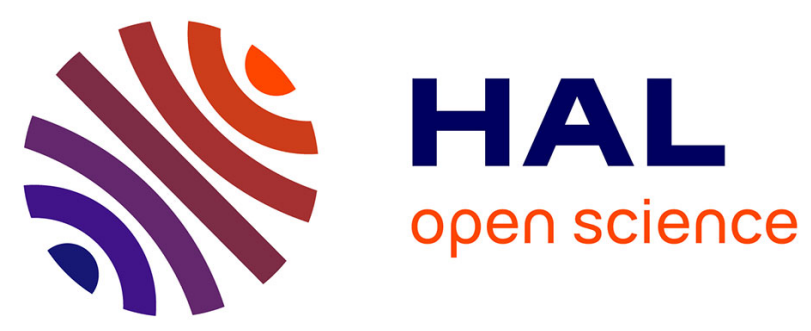

\title{
Politique des intérêts et patrimonialisation des politiques publiques en Europe du Sud: le cas des télécommunications
}

Emmanuel Négrier

\section{To cite this version:}

Emmanuel Négrier. Politique des intérêts et patrimonialisation des politiques publiques en Europe du Sud: le cas des télécommunications. Pôle Sud - Revue de science politique de l'Europe méridionale, 1996, L'Europe du Sud est-elle en retard ?, 5, pp.99-116. 10.3406/pole.1996.950 . hal-02470664

\section{HAL Id: hal-02470664 \\ https://hal.umontpellier.fr/hal-02470664}

Submitted on 23 Apr 2020

HAL is a multi-disciplinary open access archive for the deposit and dissemination of scientific research documents, whether they are published or not. The documents may come from teaching and research institutions in France or abroad, or from public or private research centers.
L'archive ouverte pluridisciplinaire HAL, est destinée au dépôt et à la diffusion de documents scientifiques de niveau recherche, publiés ou non, émanant des établissements d'enseignement et de recherche français ou étrangers, des laboratoires publics ou privés.

\section{(ㅇ)(1) $\$$}

Distributed under a Creative Commons Attribution - NonCommercial - NoDerivatives 44.0 


\section{Politique des intérêts et parimonialisation des politiques publiques} en Europe du Sud : le cas des télécommunications

Mr Emmanuel Négrier

\section{Citer ce document / Cite this document :}

Négrier Emmanuel. Politique des intérêts et parimonialisation des politiques publiques en Europe du Sud : le cas des télécommunications. In: Pôle Sud, n5, 1996. L'Europe du Sud est-elle en retard ? pp. 99-116;

doi : https://doi.org/10.3406/pole.1996.950

https://www.persee.fr/doc/pole_1262-1676_1996_num_5_1_950

Fichier pdf généré le 23/04/2018 


\section{Politique des intérêts et patrimonialisation des politiques publiques en Europe du Sud : le cas des télécommunications}

par Emmanuel Négrier

CEPEL/CNRS

Pôle Sud No 5-Novembre 1996-pp. 99 à 116

Le retard des télécommunications sud-européennes pose plusieurs problèmes d'analyse et d'interprétation politique. Celui du statut global de l'Europe du Sud dans l'évolution des politiques communautaires de télécommunications et la mise en auvre des nouvelles recettes d'action publique dans ce secteur; celui ensuite de l'unité et de la diversité de l'Europe du Sud quant à la problématique de "modernisation sectorielle", à commencer par les originalités, historiques et reproduites jusqu'à nos jours, de ses modes d'organisation; celui enfin de l'explication "qualitative" du différentiel d'action publique, où il faut évaluer la portée de deux hypotheses possibles : la sur-politisation sectorielle, la patrimonialisation des politiques publiques.

Est-ce un hasard si les analyses que nous avons pu mener sur l'évolution des politiques de télécommunication en Europe du Sud n'ont que très peu envisagé la question sous l'angle du retard (Négrier et al.1993, 1994 ; Négrier, 1994) ? D'une part, la notion même de retard, comme il est souvent dit dans ce numéro, suscite pour le politiste quelque embarras. Si l'on peut s'accorder sur le fait de ne pas prendre la statistique comme une manière satisfaisante de répondre à la question, en ce qu'elle n'explique pas les phénomènes qu'elle décrit, demeure la question de la pertinence socio-politique d'une telle interrogation. Dans ce domaine comme dans bien d'autres, il s'agit alors de se situer dans la controverse trentenaire entre développementalistes et différentialistes, où les premiers, assumant le retard (ou l'archaïsme, le traditionalisme plutôt) cherchent à en expliquer les causes, sans toujours convaincre quant à leurs présupposés, quand les seconds, réfutant la notion, se privent souvent de situer les politiques ou les systèmes qu'ils observent dans une perspective comparative dynamique. Or l'Europe du Sud, dans le domaine qui nous intéresse, est un théâtre assurément fertile de comparaison, et d'appréciation critique des thèses tenues pour dominantes sur le retard chronique vis-à-vis du Nord.

Le premier problème à résoudre est bien sûr celui du regard. L'approche socio-économique dominante fait reposer le retard du Sud sur une batterie d'arguments, parmi lesquels on trouve les données statistiques régulièrement fournies par les instances officielles de l'OCDE, de l'UIT (Union Internationale des Télécommunications), de la Commission Européenne et des centres d'études spécialisés. Ces données confirment un différentiel substantiel entre le développement des infrastructures 


\section{Pôle Sud $N^{\circ} 5$}

et services de télécommunication au Sud,par rapport au Nord. Le nombre de lignes par habitant, le taux d'équipement des ménages, le taux de numérisation des réseaux sont parmi les critères quantitatifs les plus établis pour qualifier ce retard. Le temps moyen de raccordement, la disponibilité de lignes spécialisées, l'état d'avancement des réseaux câblés, la "qualité" rapportée au temps de réaction en cas de panne sont des mesures fiables du degré de modernité des télécommunications nationales. Dans la majeure partie des cas, les pays d'Europe du Sud sont dans une position inférieure à leurs homologues du Nord. Longtemps considéré comme un problème mineur, le retard des télécommunications sud-européennes a pris une nouvelle dimension dès lors que sont apparus de nouveaux enjeux politiques :

- La capacité d'innovation des systèmes de télécommunication, dans une période de bouleversement technologique. Les politiques nationales ont commencé, au début des années 1980, à être systématiquement passées au crible de leur efficience dans l'intégration, voire l'anticipation des mutations couvertes par l'expression "nouvelles technologies de communication" : intégration des différents modes de communication en une seule et même technologie : les réseaux large-bande; modification des modes de transports dominants des messages : numérisation des réseaux ; extension des formes de communication : réseaux câblés, télématique, téléphonie portable et cellulaire...

- La globalisation des échanges, entendue comme double phénomène d'ouver- ture des échanges économiques à de nouveaux marchés (ex-pays de l'Est, Amérique latine, Extrême-Orient, etc.) et d'ouverture aux échanges concurrentiels de secteurs précédemment "régulés" (services financiers, télécommunication notamment), repose largement sur le potentiel de ces nouvelles technologies de communication. Ce secteur a donc été l'un des plus systématiquement soumis à la pression dérégulatrice, avec ses conséquences sur l'organisation techno-économique des réseaux, et notamment la remise en cause de la péréquation des services et usages sociaux des télécommunications.

- En corollaire, la capacité des systèmes institutionnels à intégrer les nouvelles normes réglementaires et d'organisation des télécommunications, telles que les préconisaient les tenants d'une équation nouvelle : innovation technologique + intégration européenne = libéralisation et privatisation. Cette équation reposa sur un retour critique sur la notion de monopole naturel, par laquelle l'économie des réseaux avait cru fonder en économie (et non en politique) l'illégitimité des systèmes privés dans les secteurs-réseaux. À l'échelle européenne, sur laquelle nous reviendrons plus loin, les monopoles, dénaturalisés, ont donc été soumis à de nouveaux critères de justification, parmi lesquels leur respect des règles du marché concurrentiel.

Le retard des télécommunications sudeuropéennes ne devient un problème politique qu'à la condition que soient établis pour souhaitables ces nouveaux objectifs, globaux et sectoriels, et imaginées les normes et stratégies générales pour les 


\section{Politique des intérêts en Europe du Sud}

atteindre ${ }^{1}$. Ce regard neuf ${ }^{2}$ posé sur la réalité économique et politique du Sud pose à son tour plusieurs problèmes ${ }^{3}$, qui feront l'objet de notre développement :

- Celui du statut global de l'Europe du Sud dans l'évolution des politiques communautaires de télécommunications. Il est d'autant plus intéressant à préciser que l'Union européenne est devenue, sinon le régulateur, du moins le porte-voix dominant des nouvelles recettes d'action publique dans ce secteur.

- Celui de l'unité et de la diversité de l'Europe du Sud quant à la problématique de "modernisation sectorielle", à commencer par les originalités, historiques et reproduites jusqu'à nos jours, de ses modes d'organisation.

- Celui enfin de l'explication "qualitative" du différentiel d'action publique, où nous évaluerons la portée de deux hypothèses possibles : la sur-politisation sectorielle, la patrimonialisation des politiques publiques.

\section{L'Europe du Sud comme espace d'adaptation communautaire}

Situons-nous d'abord dans la problématique d'intégration par les pays d'Europe du Sud des nouvelles recettes d'action publique impulsées ou traduites dans la sphère communautaire. Celles-ci, rappelons-le, reposent sur la transformation générale d'un secteur en situation de monopole public en un secteur concurrentiel, ce qui suppose, sur le plan institutionnel, un certain nombre de changements, parmi lesquels : l'établissement de règles de concurrences dans des domaines de monopole, la séparation des activités poste et télécommunication, la séparation des activités d'opérateur de celles de réglementation, et l'ouverture à la concurrence des marchés d'équipement, des services et réseaux de télécommunication, y compris le téléphone de base, dès 1998 .

En échange d'un tel bouleversement, qui affecte les formes organisationnelles et les principes d'action dominants (notamment : le service public dit à la française), la définition européenne d'un service universel est censée compenser les probables effets d'inégalité induits par le jeu du marché

Dans cette nouvelle matrice intellectuelle des politiques de télécommunication, les pays d'Europe du Sud n'ont d'abord joué qu'un rôle mineur, pour trois séries de raisons :

- Les nouvelles recettes correspondent d'abord à des situations économiques et technologiques d'abondance de réseaux et services. C'est, dans la plupart des cas, l'annonce de la saturation en réseaux et services qui permet de comprendre l'ouverture des champs nationaux à de nouvelles perspectives. Qu'elle ait débouché sur des déconvenues nationales, comme dans le cas du Plan câble français, ou sur de brutales mutations réglementaires comme dans le cas du Royaume-Uni, cette ouverture rencontre d'abord l'intérêt d'organisations et de gouvernements ayant à développer une filière au delà de ses frontières traditionnelles. Les pays d'Europe du Sud, dans ce domaine, sont tous beaucoup plus loin que leurs homologues du Nord du seuil de saturation. 


\section{Pôle Sud $N^{\circ} 5$}

- Lorsque les intérêts nationaux du Sud interviennent à l'échelle européenne, il le font sous deux formes : l'une est celle de la protection-résistance vis-à-vis de la dynamique dérégulatrice de la Commission, comme l'illustre (mais en compagnie ici de la France qui y joua un rôle charnière) le contentieux sur l'utilisation par la Commission de ses pouvoirs réglementaires directs pour ouvrir le secteur à la concurrence (Gilles, 1995 ; Simon, 1994) ; l'autre est celle de la pression en ordre dispersé : les firmes italiennes, espagnoles notamment interviennent à l'échelle européenne dans une optique de représentation de leurs propres intérêts. Les organisations publiques, comme on le verra, sont en outre composées de plusieurs institutions, aux statuts divergents, ce qui accroît d'autant le coût d'une représentation unitaire d'un projet politique identifié. Le cas de l'Italie est à cet égard emblématique (Négrier, 1997).

- Comme conséquence de ces deux premiers facteurs, les porte-voix nationaux du réformisme sectoriel sont le plus souvent soit à la périphérie du système - les professori italiens décrits par E. Brenac (1995) soit dans une situation de fragilité liée à leur manque d'ancrage historique (la DGTel espagnole, instance de régulation étatique du secteur, née au milieu des années 80 ), ou à leur instabilité politique (le gouvernement Mitsotakis jusqu'en 1994 en Grèce).

Ces trois conditions permettent de comprendre une situation politique de dépendance/résistance/opportunisme de la part des acteurs européens du Sud, qui explique à son tour que les intérêts sud-européens des télécommunications, pris globalement, n'aient pas joué de rôle central dans la construction intellectuelle des nouvelles normes d'action publique européennes et nationales.

Ensuite, la mise en œuvre de la politique communautaire s'est efforcée d'intégrer ce différentiel de développement, et cela de deux manières qui officialisent l'idée d'un retard à combler : une politique de libéralisation et une politique de développement des infrastructures et services.

La politique de libéralisation à deux vitesses a permis aux pays du Sud, du moins en grande partie, de bénéficier de moratoires dans l'adaptation de leurs contextes aux nouvelles règles de compétition. Un tel mécanisme pouvait avoir des effets pervers. Permettre aux opérateurs et industriels du Sud d'éloigner les perspectives de confrontation brutale avec leurs futurs concurrents, eux-mêmes rodés aux nouvelles règles dans le même laps de temps, pouvait, au contraire de l'effet escompté d'égalisation des chances, aggraver la fracture économique Nord-Sud, et ne laisser comme seule alternative à un protectionnisme désormais impossible la possibilité pour les acteurs sud-européens de monnayer, par des alliances du faible au fort, leur influence encore sensible sur les sanctuaires nationaux.

La politique structurelle de développement des infrastructures et services particulière aux pays en retard, et en grande partie situés au sud de l'Europe, est illustrée par les programmes STAR et TÉLEMATIQUE. Le premier s'est plus particulièrement focalisé 


\section{Politique des intérêts en Europe du Sud}

sur les infrastructures, le second sur les applications et services. Le bilan est contrasté et réside pour STAR dans trois domaines :

- L'effet de levier a permis à ces opérateurs d'engager une dynamique volontariste sur des zones qui n'auraient pas nécessairement été pourvues à un tel niveau; et surtout l'anticipation d'investissement que ces fonds ont autorisée, notamment dans la numérisation des réseaux.

- L'hétérogénéité des impacts du programme auprès de ses publics ciblés. Les PME, les plus directement concernées au départ, n'ont pas été toujours les principaux bénéficiaires de programmes par ailleurs contraints par le zonage spécifique : zones Objectif 1 de la CEE, en retard de développement, ce qui a notamment empêché la constitution sur sa base de réseaux de transfert de savoir-faire ou de technologies vers les régions les plus développées de la Communauté.

- La constitution d'un milieu, par ce programme, dont la configuration est par ailleurs restée très largement dépendante des cadres nationaux et infra-nationaux d'organisation des rapports public-privé. Le cas de l'Espagne, où les régions autonomes ont joué un rôle moteur dans cette organisation de milieu, en est une illustration.

Même s'il fut modeste dans son volume, comparé aux investissements totaux des opérateurs dans ces zones (entre 2 et $3 \%$ ), le programme STAR a donc joué un rôle non négligeable dans la diffusion d'une pratique d'aménagement des télécommunications à l'échelle européenne.

L'initiative de TÉLÉMATIQUE se situe dans le droit fil de STAR. Sa base de départ repose en effet sur le sentiment des limites dans lesquelles le premier programme s'était développé, et notamment de sa définition largement tournée vers les infrastructures. Le deuxième, complémentaire, est adopté en janvier 1991 par la Commission, selon une architecture à peu près identique : financement par les Fonds structurels ${ }^{5}$, opérations réservées en quasi-totalité aux régions Objectif 1 , constitution et management du programme assurés conjointement par la DG XIII et la DG XVI. L'évaluation qualitative de TÉLÉMATIQUE fait apparaître un essoufflement des dynamiques d'aménagement territorial des TIC telles qu'elles avaient été conçues au départ.

En dehors de ces deux programmes spécifiques, un certain nombre d'opérations spécifiques ont été appuyées par la Commission pour forcer l'adaptation du Sud aux nouvelles normes d'action. Le cas du Crash programme pour la Grèce est particulièrement intéressant : la réorganisation, qui vise à mettre le réseau grec au niveau des autres États membres, a impliqué les services de la Commission avec une ampleur jamais atteinte. Le Plan d'urgence a été établi en concertation étroite entre les autorités grecques et la Commission. Il a reposé sur l'application préalable du canevas institutionnel communautaire (séparation des fonctions d'exploitation et de réglementation, mise en place d'organes de conseils et de régulation, etc.).

Sa mise en ouvre a reposé sur l'intervention d'un cabinet multinational d'expertise, dirigeant lui-même un groupe de spécialistes grecs et internationaux. L'objectif 


\section{Pôle Sud $N^{\circ} 5$}

principal des études était essentiellement de "proposer des politiques spécifiques et des actions commerciales propres à faire de la Grèce un partenaire compétitif dans le secteur des télécommunications européennes" (Deriziotis, 1992). Ce Plan, son ingénierie, les compromis passés entre aménagement institutionnel et cofinancement (à hauteur de 104 millions d'écus), le recours aux multinationales de l'expertise, illustrent bien une logique d'importation de recettes institutionnelles et politiques ${ }^{6}$ qui ont eu, pendant la très forte conflictualité du dossier dans la politique grecque, une fragilité des compromis et une issue fatale lors du retour du PASOK au pouvoir en 1994 (Kosmidis, 1996).

Ces deux instruments communautaires (moratoire de mise en œuvre et politique structurelle spécifique) reposent sur une double hypothèse échangiste : la disponibilité de temps contre l'accord sur une voie unique de transformation sectorielle; l'allocation de ressources publiques additives contre la privatisation de la régulation. Il est cependant loin d'être évident que ces échanges soit égalitaires. Hors les effets pervers, potentiellement induits par les délais accordés et les subsides alloués, ces politiques ne contrôlent qu'une partie, minoritaire, des flux financiers, réglementaires et des relations économiques entre acteurs mondiaux du secteur. L'acclimatation aux nouvelles règles suppose, au moins au départ, l'acceptation d'une perte de contrôle politique national de la part des acteurs dominants (et pas seulement les gouvernements d'ailleurs) qui ne va pas de soi. De la même manière que les politiques structu- relles régionales (pour les régions Objectif 1 ) ne compensent pas les effets de développement inégalitaire des autres politiques de l'Union européenne (notamment la politique de concurrence, de recherche et développement, etc.), on peut douter de la capacité de politiques sectorielles de cohésion macro-régionale à enrayer d'autres tendances sectorielles de sens, sinon d'esprit, contraire.

\section{La mosä̈que sud-européenne des télécommunications}

Ayant assumé le fait que l'on pouvait approcher l'Europe du Sud de manière homogène, du point de vue de la scène des politiques européennes, il nous faut maintenant nous pencher sur l'économie des convergences et divergences qui marquent toute analyse comparée. Au delà de l'exercice de style, une telle comparaison fournit un certain nombre d'enseignements intéressants quant à la "modernité" comparée des systèmes institutionnels et, last but not least, certains résultats paradoxaux.

\section{Des histoires singulières}

Le développement des télécommunications italiennes doit beaucoup, dans sa structuration, à la période mussolinienne. La première orientation du régime, dès les années 20 , fut de composer un système institutionnel fondé sur une répartition zonale de concessions à des entrepreneurs privés (dont l'une, la SIP, Société Hydraulique Piémontaise, sera encore en fonction jusqu'en 1994, au moment de la fondation de Telecom Italia). De cette fragmentation initiale, qui reposait à la fois sur 


\section{Politique des intérêts en Europe du Sud}

l'idée de créer des liens structurels entre le régime et une pluralité d'industriels et sur la possibilité pour le gouvernement de les contrôler (ce qui aurait été plus délicat face à un seul monopole privé), découle une organisation plurielle où l'on ne trouve pas moins de six entreprises, aux statuts divers, chargées, chacune pour son domaine, de gérer les infrastructures et services de télécommunication. La relative carence du privé, et le rejet par le gouvernement Einaudi, in extremis à la Libération, de la solution ITT pour des motifs d'indépendance nationale, conduisit à une nationalisation pragmatique et progressive du secteur, au gré de la fin des concessions privées (jusqu'en 1958). Mais cette nationalisation, au contraire de la française de 1923, ne conduisit pas à une unification des sousensembles en une seule administration, mais opéra simplement une juxtaposition, au sein des participations d'État, de ces fragments, en leur conservant inchangé l'essentiel de leurs statuts, missions, et prérogatives. C'est sur cette fragmentation que s'est ensuite structuré l'ensemble des relations public-privé, mais aussi la compétition/coopération syndicale. La densité des liens unissant, autour de ce schéma, l'ensemble des protagonistes des politiques italiennes, explique en partie la difficulté singulière de réforme du système dans les années 1980-90.

Depuis 1892, les télécommunications grecques relevaient de la compétence exclusive de l'État. En 1926, une concession fut accordée pour huit ans à la New Antwerp Telephone and Electrical Works, qui devient bientôt une simple filiale de SIEMENS.
En 1930, le gouvernement accorde des droits exclusifs à SIEMENS-HALSKE AG pour développer et gérer le réseau urbain grec. Les régions périphériques et les zones de non-profit sont par contre gérées directement, de même que l'administration postale, par l'État. Ce n'est qu'après la seconde guerre mondiale, en 1949, que la compagnie publique grecque OTE a été établie, d'emblée séparée de l'administration postale.

La gestion des télécommunications espagnoles témoigne d'une évolution assez semblable. Entre 1924 et 1946, la compagnie TELEFONICA est une filiale d'ITT. Partiellement nationalisée par le régime franquiste, avec un statut d'emblée séparé de la Poste, la Compania Telefonica Nacional de Espana conserve cependant formellement un statut de droit privé, et l'État espagnol, actionnaire le plus important, ne détient jusqu'en 1987 que $47 \%$ des actions, puis $34 \%$, enfin $30 \%$. À cette période, la CNTE devient Telefonica de España SA. Le gouvernement Gonzalez, à cette période, soutient à la fois la réforme des règles internes d'organisation du secteur et une politique volontariste d'investissements internationaux, notamment en Amérique Latine. La séparation entre activité de régulation et opérateur intervient en 1985, par la création au sein du Ministère des transports de la Direction Générale des Télécommunications (la DGTel), soit largement avant la date butoir fixée par les nouvelles règles communautaires.

Les télécommunications portugaises sont, en 1887 , confiées par l'État à trois compagnies, en situation de monopole géographique : la Compagnie Anglo- 


\section{Pôle Sud $N^{\circ} 5$}

Portugaise pour Lisbonne et Porto, jusqu'en 1968 ; Correios e Telecomunicacoes de Portugal devient une administration d'État en 1911, et fournit le reste des services et infrastructures, jusqu'en 1922, où sa carence, financière notamment, est constatée dans le domaine international : la Companhia Portuguesa Radio-Marconi obtient la concession pour cette partie d'activités. Dès lors, le paysage des télécommunications est fragmenté en trois opérateurs : deux publics (TLP et CTT) et un mixte (CPRM, à $51 \%$ public seulement).

Quatre caractéristiques sont à relever dans ce bref rappel historique :

- On relève partout la présence précoce d'intérêts non nationaux dans l'organisation du secteur. Que ce soit en filigrane dans le cas italien, ou plus directement dans les autres cas, le rôle joué par ces opérateurs internationaux se déroule sur une plus longue période que dans les pays du Nord, à champion et administration nationaux plus précocement ancrés dans leur sanctuaire.

- Le statut lui-même d'administration nationale ne recouvre pas la même réalité qu'au Nord. La nationalisation, lorsqu'elle s'établit, ne rompt que rarement avec le statut privé des entreprises, et elle ne concerne souvent qu'une partie seulement de la gestion du secteur.

- Eu égard aux règles qui seront établies comme les canons de la modernité sectorielle (séparations réglementation/opérateurs ; télécommunication/poste par exemple), les pays d'Europe du Sud se distinguent par certaines innovations bien antérieures parfois à leurs homologues du Nord.

- Corollaire de ce qui précède, les pay- sages publics et privés des télécommunications sud-européennes sont, notamment dans les cas italien et portugais, marqués par une fragmentation des intérêts, qui recoupe des modalités de zonage ou de partage de secteurs par type d'activité, mais aussi, dans l'exemple italien, des processus d'échange politique ( $c f$. infra).

\section{Une mise en cuvre différentielle des politiques européennes}

Considérés comme étant en retard, les pays sud-européens bénéficient de politiques spécifiques. Elles sont néanmoins appelées à mettre en œuvre les règles communes qui participent de la politique communautaire. Ce qui nous intéresse ici est de savoir dans quelle mesure on peut repérer des convergences et des divergences dans ces processus de mise en œuvre, dont on sait qu'ils constituent un moment-clef dans la construction des politiques publiques.

Ayant déjà observé qu'ils convergeaient sur le fait de ne pas participer au cœur intellectuel et stratégique de l'élaboration des règles, nous voudrions marquer quelques-uns des principaux traits de leur mise en œuvre différenciée dans ces pays.

Nous avons déjà traité du cas grec ( $c f$. supra) à travers la mise en ouvre du Crash Programme de la Commission. Concernant le processus de réforme dans sa globalité, on peut le caractériser selon trois traits principaux :

- La fragilité des soutiens politiques internes au processus de privatisation, accompagnée d'une politisation des conflits (PASOK, alors dans l'opposition, contre 


\section{Politique des intérêts en Europe du Sud}

Nouvelle Démocratie), puis d'une tentative, par le PASOK revenu aux affaires, de nouvelles voies, plus mesurées, vers la privatisation.

- La forte capacité de mobilisation des syndicats, fortement hostiles à toute dérégulation. Le projet du PASOK de privatiser $49 \%$ seulement d'OTE échoue. La seule cotation en bourse de $6 \%$ de son capital, ce qui était possible sans réforme législative ou constitutionnelle, vaut au gouvernement une grève de quatre jours. Le management lui-même demeure globalement hostile à la privatisation, malgré les efforts consentis dans sa direction par le gouvernement.

- La capacité d'intérêts industriels à maintenir l'économie des relations institutionnelles en l'état, et notamment à résister efficacement contre tout recours à un partenariat stratégique extérieur de la part d'OTE.

Le cas espagnol est quasiment à l'opposé. Le gouvernement parvient à assurer une régulation autonome du secteur, un retrait pragmatique de l'État dans le capital de Telefonica, et l'efficience remarquable de cette dernière dans les alliances et marchés internationaux. La mobilisation en faveur de la modernisation intègre même à sa manière la structure autonomique, en conférant à la Catalogne un certain rôle dans les politiques régionalisées d'investissement et dans les forums européens de politiques publiques. Le maire de Barcelone est ainsi le représentant espagnol au sein du groupe Bangeman, chargé de mettre sur pied la politique européenne liée à la Société de l'Information. Trois traits caractérisent la politique espagnole :
- Une logique incrémentale de réforme, à partir de la réglementation initiale de 1987, et la modification de 1992 permettant d'intégrer les normes européennes. La mise en ouvre d'un Contrat de Plan EtatTelefonica, l'installation de la DGTel, la mise en place d'un Conseil consultatif réunissant les intérêts principaux du secteur permettent l'ajustement constant des positions respectives.

- Une dynamique consensuelle, dans la mesure où il n'existe pas de conflit majeur entre PSOE et PP dans ce domaine. Il n'en existe pas davantage entre le gouvernement et le management de Telefonica, globalement favorable à la réforme. Cette dernière, au contraire de bien d'autres pays européens, maîtrise une part considérable du secteur, jusqu'aux industries d'équipement. Les alliances internes et internationales s'intègrent donc généralement bien dans ce dispositif.

- L'opposition des syndicats est principiellement forte quant à l'idée de privatisation. Cependant, comme en Italie, elle est considérée comme un processus inévitable et conduit les représentants à préférer une conduite pragmatique de négociation sur les problèmes touchant davantage les conséquences (la gestion sociale) de la privatisation plutôt que sa propre mise en œuvre. Les négociations sur les retraites constituent, comme en Italie et au Portugal, une des modalités d'échange politique propres à l'univers syndical.

La mise en ouvre des réformes portugaises emprunte largement au modele espagnol, alors que la structuration du secteur est a priori plus proche de l'Italie. La frag- 


\section{Pôle Sud $N^{\circ} 5$}

mentation institutionnelle entre opérateurs, les contraintes constitutionnelles supposaient un processus en plusieurs étapes lourdes de difficultés et de négociations délicates, tant sur le plan financier que du point de vue des relations sociales. L'installation d'une instance autonome de régulation, en 1989, est précoce. La refonte de l'ensemble des compagnies en une seule intervient en 1994, avec pour objectif de rendre la privatisation plus attractive. Ce processus témoigne de trois caractéristiques générales:

- Le consensus des deux principales forces politiques gouvernementales, le Parti Socialiste et le Parti Social-Démocrate, dont l'accord est rendu nécessaire en 1989 pour réviser la constitution. Celle-ci ne permettait pas en l'état de procéder à une privatisation dans ce secteur.

- La capacité de l'instance de réglementation autonome (l'Institut des Communications du Portugal) à jouer son rôle de régulation du secteur, appuyée par le consensus politique, le soutien du management des entreprises, et une position des syndicats comparable à l'Espagne.

- Une hostilité syndicale de principe, qui en pratique ne débouche que sur une crispation sur des questions de mise en œuvre : les retraites, et l'acceptation fataliste du processus en cours.

Le processus de réforme italien est sans doute le plus complexe des quatre. D'une part, la fragmentation, d'abord géographique puis par blocs plus ou moins étanches d'activités, posa des problèmes douloureux de refonte. Au delà de cette circonstance, la dynamique des intérêts demeure longtemps très défavorable à toute velléité de réforme. Parce que sur cette fragmentation se sont établis de solides processus d'échange :

- Entre les différents industriels et les différents opérateurs, qui explique par exemple le maintien durable d'une administration d'État, l'ASST, comme opérateur à côté de l'opérateur public dominant, la SIP. Cette administration était en effet l'une des "chambres de compensation" du progrès technologique, en permettant aux fournisseurs d'écouler des stocks d'équipements dépassés à des prix sans commune mesure avec le marché. L'affaire de la vente par Olivetti d'appareils de télex dans les années 1980 est un bon exemple. L'ASST pratiquait ainsi une répartition par quotes-parts de marché dont les pourcentages sont demeurés inchangés pendant plus d'une décennie, entre plusieurs opérateurs italiens et étrangers (parmi lesquels Phillips, Siemens, Alcatel, Italtel et Olivetti) ${ }^{7}$. C'est sans doute ce qui explique la durable absence, dans le débat sur la réforme, de la Confindustria. Celle-ci représentait en effet à la fois les industriels du secteur, globalement favorables au status quo, et les intérêts économiques a priori plus sensibles à une dynamique de refonte du système public.

- Entre les forces politiques, les industriels et les forces syndicales : le syndicalisme des télécommunications reposait en partie lui-même sur cette fragmentation, avec le syndicat démocrate-chrétien (la CISL) hégémonique au sein de l'ASST, alors que la CGIL dominait dans les autres branches publiques.

La réforme, consistant en la réunion de l'ensemble des opérateurs en un seul sous la 


\section{Politique des intérêts en Europe du Sud}

houlette de Telecom Italia, a ainsi pris plus d'une décennie au cours de laquelle les projets et tentatives n'ont pas manqué (Giacalone, Vergnano, 1991). Linstallation d'une instance autonome de régulation a suivi le même parcours semé d'embûches. Les débats en cours sur la privatisation de la STET (holding public coiffant notamment les activités de télécommunication) montre, en apparence, l'essoufflement des capacités de freinage de cette coalition d'intérêts ( $c f$. infra). Le rôle d'agitateur d'idées qu'ont eu un certain nombre d'experts - les professori, parmi lesquels on doit au premier chef ranger l'actuel Président du Conseil Romano Prodi, mais aussi des journalistes, des consultants (Négrier et al., 1996) -, les conséquences de l'écroulement gouvernemental et parlementaire de la Démocratie Chrétienne, certaines révélations issues du processus Mani Pulite sont parmi les causes généralement avancées pour expliquer l'ouverture actuelle d'une nouvelle "fenêtre d'opportunité". En résumé, la situation italienne se distingue par : une fragmentation sectorielle rendant complexe toute tentative de réforme globale; une densité sociale et politique des échanges reposant sur cette fragmentation, renforçant les contraintes face au changement ; une politisation apparente de la direction sectorielle, où les positions sont d'ailleurs inversées vis-à-vis des clivages traditionnels : la Démocratie Chrétienne plutôt hostile à la libéralisation et le PDS plutôt favorable à un tel processus.

On retiendra de ce second tableau comparé les trois enseignement suivants :
- L'évolution la plus contemporaine des politiques publiques doit largement à des facteurs de longue durée. La mise en œuvre des politiques communautaires, au delà de leurs propres caractérisations du Sud pris globalement, en est largement dépendante. Elle permet de voir le Sud comme une scène spécifique de comparaison plutôt que comme un espace politique homogène.

- Parmi ces facteurs de longue durée, la fragmentation des intérêts, considérée souvent comme une donne de base de l'Europe du Sud (Schmitter, 1995), ne se vérifie pas de façon systématique. On peut la voir particulièrement à l'œuvre dans les cas portugais et italien, moins dans les deux autres pays. Surtout, les conséquences de cette fragmentation sur le processus d'action publique sont assez différentes : elle n'est une contrainte dirimante qu'en Italie et, dans une certaine mesure, pourrait apparaître a contrario comme une ressource au Portugal. C'est par l'observation de la profondeur de l'ancrage social de cette fragmentation dans les échanges politiques, et donc en mesurant la densité des intérêts et valeurs lui accordant une pertinence de longue durée, que l'on peut établir une relation entre fragmentation, style de politiques publiques et représentation des intérêts.

- On notera enfin que des principales variables mentionnées (institutionnalisation du changement, degré de conflictualitél consensus des partis gouvernementaux, positionnement idéologique et stratégique des syndicats, représentation des intérêts), fort peu se situent au même niveau de pertinence et dans la même position dans chaque pays. L'Europe du Sud, du double 


\section{Pôle Sud $N^{\circ} 5$}

point de vue de la genèse historique et de la mise en ouvre des nouvelles recettes européennes d'action publique, est donc comparable et dissemblable. Reste à tenter d'expliquer, au delà de la description, les raisons plus profondes de ces éléments de convergence et de différenciation interne.

\section{Deux thèses sur le retard sud- européen ${ }^{8}$}

Que l'on en accepte ou non les présupposés, l'analyse du retard du Sud pose un problème crucial d'explication. Au delà même de l'interprétation pointilleuse des chiffres ou des processus institutionnels, il s'agit ici de tester deux explications possibles de la spécificité du Sud. La première met l'accent sur la politisation des intérêts et des politiques comme critère de distinction. La seconde met en lumière une spécificité du rapport État-société, que l’on peut caractériser par la patrimonialisation des politiques publiques.

\section{L'étrange politique généralisée}

Lorsque le juge Green s'apprêtait à déclencher le démantèlement d'ATT aux États-Unis, il se lança dans une série de consultations afin d'observer la manière dont les différents pays développés géraient leur secteur des télécommunications. À ce titre, il rencontra Carlo Cerutti, administrateur délégué et vice-président de la STET, à qui il confia sa perplexité devant l'incroyable complexité du système italien. Afin d'expliquer la manière dont on pouvait trouver ses repères dans l'écheveau des struc- tures et officines, ce dernier n'eut qu'une simple réponse: "Siamo tutti amici!".

Cette petite anecdote, typiquement italienne, donne un éclairage très particulier sur les conditions de régulation du système fragmenté décrit plus haut. Dans le cas de l'Italie, le schème de la politisation est souvent considéré comme central. Il recouvre une partie de la réalité analysée ici dans un premier temps : la fragmentation des intérêts organisés irait de pair avec la pénétration des clivages politiques et partisans en leur sein. Ce serait même une des caractéristiques plus générales des politiques en Europe du Sud. Nous voudrions discuter ici, dans le cas particulier des télécommunications, le bien-fondé de cette hypothèse d'une "politisation généralisée de l'activité sociale".

Elle recouvre d'abord un certain nombre de réalités empiriquement observables. Le poids historique de la Démocratie Chrétienne à la tête de l'ensemble des institutions du secteur est un premier fait incontestable. La progressive admission, dans les années 70 et 80 , de certains représentants des partis laïcs et socialiste (au moment du pentapartito), renforce l'enrôlement des télécommunications dans la logique de lottizzazione : les administrateurs délégués de ces partis permettent d'élargir la base d'un profond consensus sur l'organisation globale du secteur. Au même titre que les banques, la RAI, les industries mécaniques ou l'administration, les télécommunications fournissent un cadre idéal à cette analyse en termes de politisation. L'observation des carrières à la tête de l'IRI montre que cette politisation 


\section{Politique des intérêts en Europe du Sud}

remonte loin, à l'origine d'un secteur conçu par les fascistes comme para-public : contrôlé par le Parti National Fasciste au départ, l'IRI sera ensuite dominé par les partis républicains, de telle sorte que le champ politique est devenu central puisqu'il est le lieu majeur de la distribution des emplois et de sélection-reproduction des dirigeants de l'économie (Dormagen, 1996).

La pénétration des clivages partisans à l'intérieur du système syndical des télécommunications tend à donner une touche finale à un processus qui paraît dès lors incontournable. Pourtant, cette thèse nous paraît contradictoire avec certains aspects essentiels de la conduite des politiques italiennes de télécommunication. Elle est d'abord contradictoire avec le fait que la succession de micro-alternances au sein de la Démocratie-chrétienne et du gouvernement n'ai jamais réellement constitué un élément de changement du système. La remarquable permanence des mêmes hommes à la tête des cinq sociétés, puis de Telecom-Italia et de la STET, montre un décalage certain entre système politique et élite sectorielle. Elle est ensuite assez contradictoire avec l'absence quasi-généralisée d'expertise partisane dans ce domaine. $\mathrm{Ce}$ qui est vrai pour la Démocratie-Chrétienne l'est encore plus pour les autres partis. C'est ce qui explique le rôle, très important, qu'ont joué les experts (professeurs, journalistes spécialisés, consultants) dans la construction intellectuelle des problèmes et l'invention de recettes nouvelles d'action publique.

De sorte que le rôle joué, concrètement, par les partis dans l'orientation politique du secteur nous paraît secondaire vis-à-vis de la fonction essentielle de conduite qu'exerce une élite resserrée, d'une scuola dont sont issus tous les dirigeants réels des télécommunications italiennes depuis plus de deux décennies. Infra-partisane dans sa formation, ce groupe s'est appuyé sur une pluralité de soutiens : internes à la DC (voire de l'Opus Dei, du Vatican), au sein du système économique privé, de l'État, de certaines parties du monde syndical, etc. Entre cette pluralité de partenaires, un système d'échange politique sur le long terme permettait de sécuriser les arrangements : les marchés pour les industriels, la représentativité et les conquêtes sociales pour les syndicats, les recettes budgétaires pour l'État, des postes de prestige pour les partis, et la reproduction de la légitimité des dirigeants pour la scuola.

Celle-ci bénéficiait en outre d'une forte capacité de cooptation due au fait que, au contraire de la situation française par exemple (Giraud, 1987), il n'existe par de lieu de formation unique, centrale et prestigieuse pour cette élite.

De la sorte, c'est cette élite resserrée qui a conduit, certains diront pour elle-même, la régulation du système ("siamo tutti amici") et notamment l'adaptation idéologique aux nouveaux contextes technologiques, économiques et politiques.

On peut soutenir la thèse de la politisation sur le plan formel (occupation de postes, pénétration partisane dans les institutions publiques et sociétés du secteur, etc.), et sur le plan des processus (logiques d'échange politique entre une pluralité 


\section{Pôle Sud $N^{\circ} 5$}

d'acteurs intéressés et légitimes). En revanche, il est plus difficile de la repérer sur un plan plus substantiel. La "politisation" des politiques reste un angle mort de l'analyse. La meilleure preuve de cette absence de politisation substantielle de dessein sectoriel, comparable à l'économie du grand projet typique du néo-colbertisme français (Cohen, 1992), sera sans doute apportée par les événements en cours après la victoire du pôle de l'Ulivo en avril 1996": le renversement anticipé par l'élite de la STET et de son dirigeant principal, Ernesto Pascale, des stratégies suivies jusqu'à présent ${ }^{9}$ et la convergence totale affichée désormais par ce dernier avec les thèses libérales prônées par l'Antitrust italienne dirigée par Giuliano Amato.

Une telle déconnexion entre ces trois formes de politisation (formelle, processuelle, substantielle) est liée à la nature particulière de la délégation politique à ce groupe dirigeant. On peut formuler l'hypothèse que le secteur des télécommunications représente, pour la classe politique proprement dite, un investissement coûteux pour une rentabilité aléatoire. Que cette classe ait donc massivement déserté l'expertise dans un secteur qui, malgré certaines crises d'ajustement, fournit, comme ses homologues européens, un revenu régulier à l'État. Qu'enfin les partis politiques aient, en échange de quelques rentes symboliques et matérielles, délégué la régulation du système à un groupe particulier, et par là même renoncé à conduire réellement ces politiques.

L'exemple italien est utile pour discuter en profondeur la thèse univoque de la politisation. Celle-ci est d'ailleurs avancée comme explication bien au delà de ce seul pays, comme le montre le cas de la Grèce. L'insatisfaction ressentie à l'égard de cette thèse tient bien sûr au fait qu'elle relève souvent, au Sud, d'un registre stigmatisant. Derrière le rôle déterminant accordé aux politiques peuvent aussi se révéler des mécanismes sociétaux plus profonds.

\section{La patrimonialisation des poli- tiques publiques}

L'autre thèse que nous voudrions brièvement présenter repose, au contraire de celle de la politisation, sur une analyse de plus long terme. Autour des notions de patrimonialisme (Ritaine, 1995) ou néo-patrimonialisme (Magone, 1996), la perspective est de réinsérer les politiques publiques sud-européennes dans une problématique historique des relations État-société. Celleci suppose cependant que soit dépassée l'une des interprétations weberiennes de la patrimonialité (des charges) conçue, dans l'analyse de la bureaucratie, comme un stade pré-moderne. Appliquer ce modèle aux relations sociétales reviendrait à consacrer une conception simpliste du retard. Il s'agit au contraire de tenter de faire de la patrimonialisation l'une des conditions spécifiques de la modernisation au Sud. Selon J. M. Magone, les arrangements néopatrimoniaux viennent altérer les principes de base du marché politique (distinction État-société, alternance, rationalité économique, égal accès au marché politique, etc.). La fragmentation de l'économie, 


\section{Politique des intérêts en Europe du Sud}

l'hétérogénéité sociale et politique, la colonisation de l'État par des groupes apparaissent comme éléments essentiels de ces systèmes politiques. Ce sont les conditions de l'importation de nouveaux systèmes politiques de marché qui interagissent avec l'historicité des régimes politiques. E. Ritaine, lorsqu'elle applique le modèle de l'anamorphose de l'État-Nation construit par S. Pallida (1992) traite d'une combinaison de réalités institutionnelles de l'État rationnel et de pratiques contraires (norme/illégalité, nation/particularismes, formel/informel). Elle rend compte de l'impossibilité d'établissement d'un État rationnel en l'absence de forces pouvant porter un projet structuré à même de produire un primat de l'intérêt collectif sur les intérêts particuliers. L'adaptation des structures officielles à ces relations politiques, sociales, industrielles réelles et la pratique plus systématique de l'arrangement avec les règles du jeu démocratiques sont la conséquence de cette combinatoire.

Revenons maintenant aux principales caractéristiques de l'organisation des télécommunications en Europe du Sud :

- Dans tous ces pays, là où les entreprises auxquelles a été confié le monopole, ont pour la plupart un statut privé, qu'elles ont généralement conservé en étant, tardivement et incomplètement, intégrées à l'État. Pour certains, l'explication serait à rechercher dans une histoire de très longue durée, et notamment dans le fait que, dans ces pays, la Contre-Réforme a empêché durablement la naissance d'une politique économique fondée sur le mercantilisme. Les féodalités industrielles, dues au départ à l'impossibilité pour un entrepreneur d'une autre confession de pénétrer le marché, se seraient consolidées par la non intervention de l'État en matière de concurrence (Pospischil, 1994).

- Dans la phase de monopole public, le poids des groupes privés s'analyse de façon spécifique par rapport au Nord : il pénètre en profondeur l'État et fige les perspectives de réforme en Italie. Il s'impose à l'État espagnol dans une relation dyadique. Il parvient à écarter les perspectives de pénétration étrangère stratégiques en Grèce. Il est au principe de la fragmentation institutionnelle au Portugal. Sa force est donc notablement plus importante qu'au Nord, dans son rapport à l'État et à l'intérieur de celui-ci ; son mode relationnel aussi. On pourrait être tenté d'inverser les modèles français et italien : l'histoire d'Alcatel est celle d'une couveuse publique dont le résultat est aujourd'hui l'autonomisation relative d'une entreprise privée mondiale. La puissance de l'entreprise est indissociable d'une vocation d'intérêt collectif au départ : le grand projet industriel, l'arsenal. L'histoire d'Italtel est au contraire celle de l'intégration progressive dans l'orbite publique d'une entreprise contrôlée au départ par Siemens, et qui doit composer pendant tout son parcours national avec une pluralité de concurrents, nationaux et étrangers. Dans ce cas, la puissance publique est l'expression même et la conséquence de l'influence privée, alors que l'entreprise privée française est l'expression et la conséquence de la puissance publique.

Autrement dit, bien qu'au Sud, comme au Nord, existent des traditions d'organisation en monopole, des institutions formelles 


\section{Pôle Sud $N^{\circ} 5$}

dont on peut rapprocher à gros traits le rôle sectoriel, des règles juridiques assez semblables, elles n'obéissent pas à la même dynamique des relations Etat-société. Nous qualifions le rôle différentiel du privé comme statut et comme ensemble de valeurs, de patrimoines. Au delà, la patrimonialisation des politiques publiques signifie également l'importance supérieure accordée à des logiques d'appropriation par des groupes particuliers, dans et par l'action publique, vis-à-vis de formes plus abstraites de l'intérêt collectif. Les pratiques clientélistes dans les relations Parti-OTE en Grèce, la reproduction de la scuola à la tête des télécommunications italiennes, pour n'être pas directement liées à une appropriation de l'État par des intérêts industriels privés, relèvent pourtant de ce processus où prévaut, sur le long terme, un intérêt fragmentaire, semi-collectif, sur une politique d'intérêt général. La politisation ne serait qu'un phénomène visible et partiel d'un processus plus fondamental de "privatisation" des intérêts obéissant à une logique patrimoniale. Se trouve alors posé le problème de la capacité de processus de patrimonialisation à produire et reproduire biens publics et services collectifs. L'état des télécommunications en Europe du Sud n'est ni totalement patrimonial, ni légalrationnel; il est un compromis entre les deux. La nature de ce compromis, qui découle de l'analyse des échanges politiques auxquels il donne lieu, peut parfaitement déboucher sur une grande modernité sectorielle ou au contraire sur des blocages, conjoncturels ou structurels, dans le processus de modernisation. L'Espagne et le
Portugal illustreraient assez bien le premier cas, l'Italie et la Grèce le second. Dans une telle situation, la "réussite" du processus de modernisation ne passe pas par l'importation de recettes standardisées, comme l'a bien démontré l'échec du Crash Programme européen en Grèce sur ce point, ni par une quelconque imitation de modèles, que l'on a cru pouvoir appliquer, en Italie, en suivant l'exemple anglais. Elle passe souvent par la confortation de groupes qui, pour être hétérodoxes vis-à-vis des normes tenues au nord pour excellentes, sont néanmoins des médiateurs obligés, et souvent efficients, du changement. L'un des ultimes paradoxes de ce processus en marche est de donner à l'État une capacité inédite de pilotage de l'européanisation de l'action publique, ce même Etat dont on a tant dénoncé au Sud la carence, l'incomplétude ou la captivité vis-à-vis des intérêts socioéconomiques.

À quelles conditions peut-on fonder la modernisation sur une économie patrimoniale des échanges politiques? En avançant cette question en ultime remarque, nous voulons souligner que le problème du retard et des recettes pour sa réduction ne sont qu'un enjeu parmi d'autres des politiques sectorielles en Europe du Sud et, partant, en Europe communautaire. Les fragmentations d'intérêts, qui sont sans doute à l'origine d'une modernisation à géométrie variable (il suffit de mentionner les avancées italiennes en matière de téléphonie portable, ou celles espagnoles en matière d'investissements internationaux), mettent davantage en question le mérite respectif des processus qui prétendent à son 


\section{Politique des intérêts en Europe du Sud}

accomplissement. Du reste, la communautarisation des télécommunications n'engendre-t-elle pas une mosaïque de situations et de cultures nationales telles que la régulation de l'ensemble européen repose elle aussi sur une part de cette patrimonialisation trop vite rangée au rayon des archaïsmes politiques?

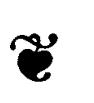

\section{Notes}

1. Pour le dire autrement, "il y a problème quand des acteurs sociaux perçoivent des écarts entre ce qui est, ce qui pourrait être ou devrait être. Il s'en suit un processus de classement qui fait intervenir une qualification comme relevant de la sphère de compétence des autorités publiques" (Padioleau, 1982).

2. L'intérêt d'analyser un regard tient ici autant dans ce qu'il dénonce que dans ce qu'il dissimule à son tour. Le problème du stigmate, c'est l'astigmate.

3. Il est considéré ici que la notion de retard est relativement indépendante de la notion de progrès, social notamment. L'état d'avancement institutionnel et technologique attribué à la Grande-Bretagne va de pair avec une augmentation des tarifs du téléphone de base, alors que les pays "attardés" du Sud ont pour point commun une tarification très avantageuse sur ce même créneau, qui concerne d'abord les revenus les plus modestes.

4. La nature et la portée de cette compensation font l'objet d'un débat dont nous ne pouvons ici rendre compte.

5. Financement à hauteur de $50 \%$ de l'opération lorsqu'elle comprend un partenariat privé, de $75 \%$ pour les autres, notamment dans le secteur public, selon le règlement FEDER.

6. Recettes que l'on peut ici qualifier d'européennes, à l'exception sans doute d'une certaine idée de la subsidiarité, vis-à-vis de laquelle la sous-représentation des autorités grecques dans le dispositif constitue une notable exception.

7. On sait cependant que de telles pratiques sont loin d'être le seul apanage de l'Italie, lorsque l'on prend pour exemple les effets de la "consanguinite" industrielle entre Alcatel et France Télécom sur les surfacturations d'équipements.

8. Pour des raisons de place et de disponibilité de données comparables de terrain, nous ne pouvons discuter, sauf exception, ces deux thèses en nous appuyant sur l'ensemble des pays. Nous limitons donc pour l'instant l'essentiel de l'argument à l'Italie, en précisant qu'une version de ce développement figure dans $\mathrm{E}$. Négrier, "Existe-t-il une politique italienne des télécommunications ?", à paraître dans le numéro spécial Italie de Quaderni, 1997.

9. Et notamment la résistance à toute forme de privatisation brutale, la limitation des alliances internationales visant le territoire italien aux aspects technologiques (avec IBM), la promotion d'une autorité spécifique et distincte de l'Antitrust, la mise en œuvre, préalable à la privatisation, d'un vaste plan de câblage en fibre optique à large bande visant 10 millions de foyers à l'horizon 1999. L'annonce d'une négociation avec Cable $\&$ Wireless sur un échange de participation, l'intégration de la STET dans le quatrième pôle international d'opérateurs $\mathrm{C} \& \mathrm{~W}-$ Veba-Bouygues, la privatisation annoncée et précédée d'une refonte réglementaire, la remise en question du plan de câblage au seul profit de la STET et de Telecom-Italia sont autant de revirements politiques très significatifs de l'adaptabilité de l'élite des télécoms italiennes à une contrainte environnementale qui change. 


\section{Pôle Sud $N^{\circ} 5$}

\section{Références}

Brenac E., "Néolibéralisme et politiques industrielles : les conditions et formes différenciées d'un changement de paradigme. L'exemple des télécommunications en Europe", in Jobert B., Théret B. (dirs.), Le tournant néo-libéral en Europe, Paris, L'Harmattan, 1994.

Cohen E., Le colbertisme "high tech". Economie des Télecoms et du grand projet, Paris, Pluriel/Hachette, 1992.

Deriziotis C., "Grèce : plan d'urgence pour les télécommunications", XIII magazine, n 3, octobre 1991.

Dormagen J.-Y., "Profession politique et politisation des professions en Italie : le cas des conseillers d'administration de l'IRI (1939-1990)", Communication au Congrès de l'AFSP, Aix-en-Provence, avril 1996.

Giacalone D., Vergnano F. (dirs.), “Tamburi di latta. L'Italia dei telefoni nella sfida mondiale", Il Sole, $\mathrm{n}^{\circ} 24$, 1991.

Gilles L., "La politique publique des télécommunications : service public versus service universel", in Musso P., Rallet A., Strategies de communication et territoires, Paris, L'Harmattan, 1995.

Giraud C., Bureaucratie et changement, Paris, L'Harmattan, 1987.

Kosmidis M., "A comparative analysis of EU telecommunications policy in Southern Europe : Greece, Spain and Portugal", Papier présenté au Congrès de l'ECPR, Oslo, 1996.

Magone J. M., "Patrimonialist Patterns of Political Représentation. The Logic of Party System Change in Southern Europe", ECPR, Oslo, 1996.

Négrier E., “Existe-t-il une politique italienne des télécommunications ?”, Quaderni, à paraître 1997.

Négrier E., Briole A., Lauraire R., "Des contrats sans rupture : l'émergence de la contractualisation dans les politiques publiques de télécommunication en Europe du Sud", in Gaudin J.-P. (dir.), "La négociation des politiques contractuelles", Paris, L'Harmattan, 1996.

Négrier E., "Identité européenne et territoire. Le cas des télécommunications en Europe du Sud", Rapport pour le Programme de recherche sur les sciences de la communication du CNRS (Dominique Wolton), 1994.

Négrier E., Rabaté F., Rallet A., "L'aménagement territorial des technologies de l'information et de la communication. Stratégies et prospectives d'acteurs - dynamique locales, régionales et communautaires", CEPEL, 1994.

Négrier E., Briole A., De la Torre L., Lauraire R., "Les politiques publiques de télécommunication en Europe du Sud. Service public et dynamiques territoriales des intérêts", Rapport pour le Commissariat Général du Plan, CEPEL, 1993, 4 tomes.

Padioleau J. L'État au concret, Paris, PUF, 1982.

Pallida S., "L'anamorphose de l'État-nation : le cas italien", Cahiers Internationaux de Sociologie, $\mathrm{n}^{\circ} \mathrm{XCIII}$, 1995.

Pospischil R., "L'adieu au monopole", Le Communicateur, n² 25, Printemps 1994.

Ritaine E., "Hypothèses pour le sud de l'Europe : territoires et médiations", working paper, Institut Universitaire Européen, Centre R. Schuman, Florence, 1995.

Schmitter P. C., "Groupes d'intérêts et consolidation démocratique en Europe méridionale", Pôle Sud, n 3, 1995.

Simon J.-P., "Service public, service universel”, Réseaux, n 66, Août 1994. 